Sharif University of Technology
Scientia Iranica
Transactions E: Industrial Engineering
hCIENTIA

\title{
Solving a multi-objective model toward home care staff planning considering cross-training and staff preferences by NSGA-II and NRGA
}

\author{
H. Habibnejad-Ledari ${ }^{a}$, M. Rabbani ${ }^{a, *}$, and N. Ghorbani-Kutenaie ${ }^{b}$ \\ a. School of Industrial Engineering, College of Engineering, University of Tehran, Tehran, Iran. \\ b. Department of Industrial Engineering, School of Engineering, Alzahra University, Tehran, Iran. \\ Received 4 June 2017; received in revised form 9 April 2018; accepted 4 August 2018
}

\author{
KEYWORDS \\ Home care; \\ Staff assignment; \\ Cross-training; \\ Optimization; \\ NSGA-II; \\ NRGA.
}

\begin{abstract}
Home Care (HC) staff assignment problem is defined as deciding which staff to assign to each patient. In this study, a multi-objective non-linear mathematical programming model is presented to address staff assignment problem considering crosstraining of caregivers for $\mathrm{HC}$ services. The first objective of the model is to minimize the cost of workload balancing, cross-training, and maintenance. The second objective minimizes the number of employees for each service, while the third objective function maximizes the satisfaction level of caregivers. Several constraints including skill matching, staff preferences, regularity, synchronization, staff absenteeism, and multi-functionality are considered to build a service plan. Due to NP-hardness of the problem, a Non-dominated Sorting Genetic Algorithm (NSGA-II) with a proposed who-rule heuristic initialization procedure is applied. Due to the absence of benchmark available in the literature, a Non-dominated Ranking Genetic Algorithm (NRGA) is employed to validate the obtained results. The data required to run the model are gathered from a real-world HC provider. The results indicate that the proposed NSGA-II is superior to the NRGA with regard to comparison indexes. Based on the results obtained, it is now possible to determine which staff to cross-train for each service and how to assign staff to services.
\end{abstract}

(C) 2019 Sharif University of Technology. All rights reserved.

\section{Introduction}

Home Care (HC) service defined as visiting, assisting, nursing, and delivering medical, nonmedical, and paramedical services to patients, the elderly, and disabled people at their homes was offered as an alternative to traditional hospitalization as well as to providing an opportunity for citizens to stay in their homes [14]. HC services consist of nonmedical services (such as

\footnotetext{
*. Corresponding author. Tel.: +982188021067; Fax: +982188350642

E-mail address: mrabani@ut.ac.ir (M. Rabbani)
}

cleaning, preparing meals, doing housework, bathing, dressing, and shopping) and medical services (such as nursing, physiotherapy, and occupational therapy, taking care of children, elderly, people with disabilities, and conditions treated (e.g., patients suffering from AIDS, cancer, and neurological disorders)). The main advantages of $\mathrm{HC}$ include a considerable reduction in hospitalization rate, improving the quality of life, and significant cost saving $[5,6]$.

Increasing health care costs and change in the age distribution of population have led to an increase in the number of $\mathrm{HC}$ clients, and $\mathrm{HC}$ providers, therefore, face a large number of patients $[7,8]$. At the same time, these providers have to reduce their resources to mitigate costs and, at the same time, increase their 
competitiveness. Moreover, demand is unpredictable and service duration is very volatile. Thus, they strive to achieve efficient workforce management that is necessary to increase service quality and reduce operational costs. However, in reality, designing an efficient workforce management policy is hard to attain [7]. In the literature, this problem is referred to as HC staff assignment problem, which is concerned with deciding which staff to assign to each admitted patient [1]. HC service involves dealing with a large number of patients with rapid changes in clinical and social conditions. To deal with this issue, the number of caregivers should increase. It increases service quality and leads to high patients' satisfaction and time saving [7]. However, an increase in the number of caregivers will lead to an increase in operating and human resources costs as well as idle time of caregivers, which is not economically justifiable. On the other hand, reducing the number of caregivers leads to service quality deterioration $[9,10]$. Therefore, it is recommended that their skills and abilities be risen instead of increasing the number of caregivers so that they can operate more services. This description refers to a concept called "cross-training" in the literature.

Cross-training the staff is one of the first steps in HC staff assignment [11]. Increasing cross-trained staff leads to production flexibility and is a significant goal for today's organizations $[12,13]$. Achieving this goal at organizations or department level leads to the creation of individual training goals [14]. Cross-trained staff are capable of performing multiple services. Creating cross-trained staff includes three main steps [11]: (1) hiring staff with appropriate basic skills, (2) giving them necessary off-the-job training, and (3) assigning services to them so that the required experience can be acquired and maintained. Cross-training enjoys numerous advantages such as positively affecting operational performance indicators (e.g., the throughput time), dealing with fluctuation in the supply of human resources more effectively, increasing the possibility of workload sharing, creating opportunities for job rotation, and minimizing idle time $[15,16]$.

Most of the above-mentioned advantages can be achieved without having to perform full cross-training. A full cross-trained system is advantageous only when the relative flexibility costs are low [17]. Achieving a full cross-trained system is, however, very costly and may lead to significant productivity loss due to the shift of staff between services. In addition, there are some social reasons for limiting cross-training and labor flexibility in firms. A fully cross-trained workforce makes jobs more similar, which may, in turn, impair social identity and lead to motivational deficits. It may also cause social loafing (i.e., a situation in which no one feels exclusively responsible to do the dirty work) $[18,19]$. Referring to the foregoing discussion, cross-training can be potentially motivating or demotivating based on the selected policy, which suggests that it is often better not to attempt full cross-training. This raises the following question: "who should be crosstrained for each service?".

This study addresses staff assignment problem considering cross-training of caregivers in healthcare systems to determine which staff should be crosstrained for each service and how they should be assigned to services. A multi-objective non-linear mathematical programming model is proposed, where the first objective function aims to minimize workload balancing, cross-training, and maintenance costs. The second objective function attempts to minimize the number of employees for each service, while the third objective function is concerned with maximization of caregivers' satisfaction level. The behavior of the various elements and features of the model is evaluated in a real-world HC provider as our case study. Several constraints with respect to Skill Matching (SM), staff preferences (PRE), regularity (REG), synchronization (SYN), staff absenteeism, time capacity, and multifunctionality level are taken into account to build a service plan. In addition, the chaining concept is used to balance workloads of caregivers and allow shifting jobs among caregivers. Finally, due to the cultural conditions prevailing in Iran, it is supposed that services for men and women are separately performed. For the problem being NP-hard, a Non-dominated Sorting Genetic Algorithm (NSGA-II) with a proposed heuristic initialization procedure is applied, and the proposed model is solved based on a dataset collected from our case. In the proposed initialization heuristic, a who-rule is applied to determine which staff should be assigned to services. The parameters are tuned by Taguchi method. Due to the absence of benchmark available in the literature, a Non-dominated Ranking Genetic Algorithm (NRGA) is employed to validate the obtained results. Three comparison indexes are considered, and the results show the superiority of the proposed NSGA-II to the NRGA with respect to comparison indexes.

The remainder of this study is organized as follows. Section 2 provides a brief review of the related literature. Section 3 presents problem definition as well as the assumptions in more detail. In Section 4, the parameters and decision variables are introduced, and the mathematical model is presented. Section 5 details the solution methodology and designs the NSGAII to solve the proposed model. In addition, the implementation of Taguchi method and other details proposed for parameter tuning are described in this Section. The proposed real-world case study and the relevant computational results are given in Section 6 . Finally, Section 7 is dedicated to concluding remarks and directions for future research. 


\section{Literature review}

\subsection{HC staff assignment problem}

Staff assignment problem in health care systems is an interesting topic for researchers in different fields. In the literature, optimization of different financial measures, such as personnel cost, overtime cost, outsource cost, waiting cost, and travel cost, has been considered as an objective function. Borsani et al. [3] presented an Integer Linear Programming (ILP) model for scheduling human resource as an HC staff planning problem and applied it to a real dataset collected from Italian HC providers. Akjiratikarl et al. [20] addressed scheduling of homecare workers in an attempt to minimize the distance traveled by workers. They used a Particle Swarm Optimization (PSO) algorithm to deal with this problem. Hertz and Lahrichi [21] presented a Mixed Integer Programming (MIP) model with the aim of balancing the workload of the nurses and minimizing the traveled distances. They applied a Tabu Search (TS) algorithm to handle the proposed model. Rabeh et al. [22] proposed a mixed ILP model with the objective of minimizing both the traveled and waiting times and took advantage of Lingo to solve the problem. Rasmussen et al. [1] studied Vehicle Routing Problem (VRP) with time windows in the healthcare system. They formulated it as a set partitioning problem and presented a branch-and-price solution algorithm based on the soft preference constraints to solve it.

Gamst and Jensen [23] addressed the long-term homecare scheduling problem and designed a branchand-price algorithm to solve the proposed model in an attempt to minimize the overall cost and maximize the service level. Liu et al. [24] proposed two MIP models to address VRP in home health care logistics. They considered delivery, pickup, and time windows and applied genetic algorithm and TS method to solve the presented model. Allaoua et al. [2] presented an ILP model with the aim of establishing the optimal routes and rosters for the HC staff and applied a metaheuristic based on decomposition to deal with this problem. A mathematical model was developed by Mankowska et al. [25] in an attempt to optimize performance measures concerning economic and service levels. They applied a heuristic based on a sophisticated solution representation to solve the problem. Duque et al. [26] proposed a bi-objective programming model with the aim of minimizing the distance traveled by the caregiver and maximizing the service level and designed a two-stage solution strategy to deal with it.

There are a few papers considering continuity of care and demand or uncertainty in staff assignment problem in healthcare environment. Lanzarone and Matta [5] studied the robust $\mathrm{HC}$ staff assignment problem under the continuity of care and proposed an analytical structural policy to solve this problem. They believed that robust staff planning is crucial for operations in homecare companies to increase the quality of their services. Mutingi and Mbohwa [10] designed a fuzzy simulated algorithm to address caregivers scheduling problem in homecare companies. Based on the obtained results, their algorithm has the capability to solve this problem. Carello and Lanzarone [27] proposed a cardinality-constrained robust assignment problem and analyze it on a real home care company as a case study. The results show the capability of the model to reduce nurse's overtime costs. Mutingi and Mbohwa [28] presented a fuzzy particle swarm optimization approach to staff assignment problem in healthcare environment with the aim of maximizing workload balance and minimizing violation of patients' time window. Mutingi and Mbohwa [29] addressed the caregiver planning problem and designed a fuzzy genetic algorithm to solve the problem. Their model included multiple conflicting objectives and preference constraints.

\subsection{Cross-training}

Recently, cross-training has become very important for the global business to deal with variations in product mix and fluctuations in the supply of human resources. Both in the literature and in practice, implementation of cross-training policies has been recognized to play a significant role in organizations. Bokhorst et al. [30] investigated the need for incorporating the whorule in simulation studies. They explored the flow time impact of different who-rules on dual resourceconstrained systems where workers differ from each other in terms of workload, number of skills, and task proficiencies. In another paper, Bokhorst et al. [31] presented an integer goal programming model to evaluate cross-training policies where the objective was to minimize flow time mean and standard deviation from the operation management and human resource management viewpoints. Slomp et al. [15] proposed an integer programming model for minimizing workload and cross-training costs. They attempted to answer the following question: "which worker should be crosstrained for each machine?" Yang [32] evaluated and compared a set of cross-training policies with different numbers of cross-trained workers, additional machines, and additional skills per cross-trained worker in job shops.

Yue et al. [33] studied cross-training policies in a parallel job-shop environment. They considered learning and forgetting phenomena, and assumed that new part types were frequently introduced into the system. Li et al. [34] proposed a bi-objective integer programming model in an attempt to minimize the average salary and maximize satisfaction degree. They employed a NSGA-II algorithm and compared 
its results with those of Multiple Objective Particle Swarm Optimization (MOPSO). Liu et al. [35] proposed a multi-objective model aimed at balancing total processing times and minimizing total training cost in seru production systems. They also developed a threestage heuristic algorithm to tackle the proposed model. By taking into account cross-training, the effect of worker learning and forgetting, and the heterogeneity within worker pool, Feng and Fan [36] simulated a production line in car engine parts plant and compared Work In Process (WIP), adaption time, and productivity under different cross-training policies with each other. Habibnejad et al. [37] presented a bi- objective non-linear programming model with the aim of minimizing workload balancing, cross-training, and maintenance and transportation costs and maximizing caregivers' satisfaction level. They considered several constraints such as maximum allowable distance for transportation, maximum allowable consecutive shifts, and budget capacity and solved the proposed problem with an exact algorithm.

Table 1 provides a summary of the researches reported on homecare systems according to the most common constraints and objective functions taken into account for homecare services planning in chronological order. In addition, Table 2 presents the cross-training

Table 1. Overview of the literature regarding home care.

\begin{tabular}{|c|c|c|c|c|c|c|c|c|c|c|}
\hline \multirow[t]{2}{*}{ Paper } & \multicolumn{5}{|c|}{ Objective functions* } & \multicolumn{5}{|c|}{ Constraints $^{* *}$} \\
\hline & TT & $\mathrm{CC}$ & DD & BW & SP & TW & SM & PRE & REG & SYN \\
\hline Akjiratikarl et al. [20] & & & $\sqrt{ }$ & & & $\sqrt{ }$ & & & & \\
\hline Hertz and Lahrichi [21] & & & $\sqrt{ }$ & $\sqrt{ }$ & & & $\sqrt{ }$ & & & \\
\hline Trautsamwieser et al. [48] & $\sqrt{ }$ & & & & $\sqrt{ }$ & $\sqrt{ }$ & $\sqrt{ }$ & $\sqrt{ }$ & & \\
\hline Rasmussen et al. [1] & & $\sqrt{ }$ & & & $\sqrt{ }$ & $\sqrt{ }$ & & & & \\
\hline Gamst and Jensen [23] & $\sqrt{ }$ & $\sqrt{ }$ & & & $\sqrt{ }$ & $\sqrt{ }$ & $\sqrt{ }$ & & $\sqrt{ }$ & \\
\hline Liu et al. [24] & & $\sqrt{ }$ & & & & $\sqrt{ }$ & & & & $\sqrt{ }$ \\
\hline Mankowska et al. [25] & $\sqrt{ }$ & & $\sqrt{ }$ & & & $\sqrt{ }$ & $\sqrt{ }$ & & & $\sqrt{ }$ \\
\hline Duque et al. [26] & & & $\sqrt{ }$ & & $\sqrt{ }$ & & & $\sqrt{ }$ & $\sqrt{ }$ & \\
\hline Habibnejad et al. [37] & & $\sqrt{ }$ & $\sqrt{ }$ & $\sqrt{ }$ & $\sqrt{ }$ & & $\sqrt{ }$ & $\sqrt{ }$ & & \\
\hline This study & & $\sqrt{ }$ & & $\sqrt{ }$ & $\sqrt{ }$ & & $\sqrt{ }$ & $\sqrt{ }$ & $\sqrt{ }$ & $\sqrt{ }$ \\
\hline
\end{tabular}

*TT: Time (travel, waiting, etc.); CC: Costs (assignment, scheduling, etc.); DD: Travelled distances;

BW: Balance of the workload; SP: Staff preferences.

**TW: Time windows; SM: Skill matching; PRE: Staff preferences; REG: Regularity; SYN: Synchronization.

Table 2. Overview of the literature regarding cross-training and labor assignment.

\begin{tabular}{|c|c|c|c|c|c|c|c|c|}
\hline \multirow[t]{2}{*}{ Paper } & \multicolumn{8}{|c|}{ Feature* } \\
\hline & 1 & 2 & 3 & 4 & 5 & 6 & 7 & 8 \\
\hline Stewart et al. [49] & $\sqrt{ }$ & & $\sqrt{ }$ & & & & & \\
\hline Bokhorst et al. [30] & & $\sqrt{ }$ & & & & & & \\
\hline Slomp et al. [15] & $\sqrt{ }$ & & $\sqrt{ }$ & & $\sqrt{ }$ & $\sqrt{ }$ & & \\
\hline Easton [50] & $\sqrt{ }$ & & $\sqrt{ }$ & $\sqrt{ }$ & $\sqrt{ }$ & & & \\
\hline Kim and Nembhard [51] & $\sqrt{ }$ & & & $\sqrt{ }$ & & & & \\
\hline Gnanlet and Gilland [52] & $\sqrt{ }$ & & & & $\sqrt{ }$ & & & \\
\hline Paul and MacDonald [53] & $\sqrt{ }$ & & & & $\sqrt{ }$ & & & \\
\hline Sammarco et al. [54] & & $\sqrt{ }$ & $\sqrt{ }$ & & & & $\sqrt{ }$ & $\sqrt{ }$ \\
\hline Habibnejad et al. [37] & $\sqrt{ }$ & $\sqrt{ }$ & $\sqrt{ }$ & $\sqrt{ }$ & & & & \\
\hline This study & $\sqrt{ }$ & $\sqrt{ }$ & $\sqrt{ }$ & $\sqrt{ }$ & $\sqrt{ }$ & $\sqrt{ }$ & & \\
\hline
\end{tabular}


and labor assignment features and summarizes those features addressed in the literature review. For the first time, this paper incorporates the cross-training concept in homecare staff assignment problem based on a realworld HC provider. In addition, cross-training and labor allocation using who-rule are studied simultaneously, and the most common constraints are taken into account for cross-training and labor allocation problem, concurrently.

\section{Problem definition}

Dam homecare medical center is a non-governmental organization founded in Iranian Academic Center for Education, Culture, and Research (ACECR) in 1999. It is a 24-hour medical center that provides its 11000 patients in Tehran, Iran with HC services. The process commences when a citizen applies for $\mathrm{HC}$ services, and a preadmission is initiated. Each visit commonly takes between 1 to 5 hours. The manager decides which caregiver should be assigned to each patient by taking into account a set of factors such as the patients' medical statue, caregivers' skills and training level, and the patients and caregivers' preferences.

The objectives of the organization are two-fold: (a) minimization of the number of employees for each service and costs associated with workload balancing, training, and maintenance and (b) maximization of staff preferences so that the patients' needs are met. Some constraints are required to build a service plan in Dam organization, as presented in the following:

1. Skill Matching (SM): A service requires staffs with specific skills or training;

2. Staff preferences (PRE): Staff preferences respecting time and staff assignment;

3. Regularity (REG): The number of employees for each service;

4. Synchronization (SYN): A service requires more than one staff member.

Moreover, each caregiver can be cross-trained for the maximum number of services called multifunctionality level. A caregiver's multi-functionality represents the number of services that one is able to deliver. The maximum number of working hours per day is another constraint that the organization has to take into account. Since there are enough regular working hours to cover all requirements, overtime and non-regular shifts are not involved. In this paper, the chaining concept is used to reallocate jobs among caregivers, leading to a more balanced workload that is desired from social and economic perspectives. Finally, it is supposed here that services for men and women be separately provided due to the cultural conditions prevailing in Iran.

\section{Proposed model}

In this section, the proposed multi-objective mathematical model is presented whose first objective is to balance workload and minimize cross-training and maintenance costs. In the second objective function, the number of employees for each service is minimized, while the third one attempts to maximize caregivers' preferences.

The indices and parameters used in the presented model are defined as follows:

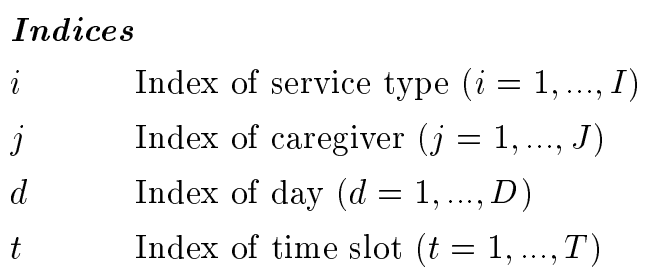

\section{Parameters}

I Number of services

$J \quad$ Number of caregivers

$D \quad$ Number of days

$T \quad$ Set of time slots

$J M \quad$ Set of male caregivers

$J F \quad$ Set of female caregivers

$M S \quad$ Set of services requiring male caregivers

$F S \quad$ Set of services requiring female caregivers

$A_{d t} \quad$ Set of absent caregivers on day $d$ at time slot $t$

$N C_{i} \quad$ Number of caregivers required for service $i$

$M_{j} \quad$ Maximum multi-functionality for caregiver $j$

$E_{i j} \quad$ Efficiency factor for service $i$ when provided by caregiver $j$

Time $_{i} \quad$ Normalized visit time of service $i$

$C_{j t} \quad$ Costs associated with caregiver $j$ 's workload deviation from the average value at time slot $t$

$T C_{i j} \quad$ Costs of training caregiver $j$ for service $i$

$M C_{i j} \quad$ Maintenance costs of caregiver $j$ for service $i$

$D_{i d t} \quad$ Demand of service $i$ on day $d$ at time slot $t$

$C P_{i j} \quad 1$, if caregiver $j$ prefers service $i ; 0$ otherwise

$K_{j} \quad$ Maximum working time of caregiver $j$ in each day

$\pi_{1}, \pi_{2} \quad$ Weight factors

$R \quad$ A large number 


\section{Decision variables}

$S_{j} \quad$ Satisfaction level of caregiver $j$

$R_{i} \quad$ Number of caregivers assigned to

$Y_{i j} \quad$ service $i$

service $i$; 0 otherwise

$Z_{i j d t} \quad 1$, if caregiver $j$ is assigned to service $i$ on day $d$ at time slot $t$; 0 otherwise

$X_{i j d t} \quad$ Number of times caregiver $j$ is assigned to service $i$ on day $d$ at time slot $t$

It is assumed here that training should lead to workload balance among caregivers in various circumstances; as a result, there is always the possibility of chaining. Equally loaded caregivers can be achieved by minimizing the caregivers' workload deviation from the average workload in different periods. Some of the services in the organization need special care due to the cultural conditions prevailing in Iran, i.e., requiring either male or female caregivers. The sets of $J M, J F, M S$, and $F S$ are defined for this purpose. $N C_{i}$ is defined due to the synchronization constraint, indicating that some services could possibly call for more than one caregiver. The cost of training caregiver $j$ for service $i$ is denoted by $T C_{i j}$ and is zero for a caregiver who is already qualified for a particular service. $E_{i j}$ is determined by the operational team. The smaller the value of $E_{i j}$ is from 1, the more efficient caregiver $j$ will be for performing service $i$. On the other hand, as $E_{i j}$ goes higher than 1, caregiver $j$ is assumed to become more inefficient in performing service $i$.

\subsection{Mathematical model}

$$
\begin{aligned}
\min z_{1}= & \pi_{1} \sum_{d=1}^{D} \sum_{j=1}^{J} \sum_{t \in T} C_{j t} \cdot\left|\sum_{i}^{I} \operatorname{Time}_{i} \cdot X_{i j d t}-\tilde{X}_{d t}\right| \\
& +\pi_{2} \sum_{i=1}^{I} \sum_{j=1}^{J} Y_{i j}\left[T C_{i j}+M C_{i j}\right] \\
\min z_{2}= & \sum_{i=1}^{I} R_{i}, \quad \max z_{3}=\sum_{j=1}^{J} S_{j}
\end{aligned}
$$

subject to:

$$
\begin{array}{ll}
\tilde{X}_{d t}=\frac{\sum_{i=1}^{I} \sum_{j=1}^{J} \text { Time }_{i} \cdot X_{i j d t}}{J} & \forall d, t \in T, \\
\sum_{j=1}^{J} Y_{i j} \leq R_{i} & \forall i, \\
Z_{i j d t} \leq R . Y_{i j} & \forall j, d, t \in T,
\end{array}
$$

$$
\begin{aligned}
& X_{i j d t} \leq R . Z_{i j d t} \quad \forall i, j, d, t \in T, \\
& \sum_{j=1}^{J} X_{i j d t}=N C_{i} . D_{i d t} \quad \forall i \notin M S, F S, d, t \in T \\
& \sum_{j \in J M} X_{i j d t}=N C_{i} \cdot D_{i d t} \quad \forall i \in M S, d, t \in T, \\
& \sum_{j \in J F} X_{i j d t}=N C_{i} . D_{i d t} \quad \forall i \in F S, d, t \in T, \\
& \sum_{i=1}^{I} \operatorname{Time}_{i} \cdot E_{i j} \cdot X_{i j d t} \leq K_{j} \quad \forall j, d, t \in T, \\
& \sum_{i=1}^{I} Y_{i j} \leq M_{j} \quad \forall j \\
& S_{j}=\frac{\sum_{i=1}^{I} \sum_{d=1}^{D} \sum_{t \in T} C P_{i j} \cdot Z_{i j d t}}{\sum_{i=1}^{I} \sum_{d=1}^{D} \sum_{t \in T} Z_{i j d t}} \forall j \\
& Z_{i j d t}=0 \quad \forall i, j \in A_{d t}, d, t \in T, \\
& S_{j}, \text { dev }_{j d t} \geq 0 \quad \forall j, d, t \in T, \\
& X_{i j d t}, R_{i} \geq 0 \text {, Integer } \quad \forall i, j, d, t \in T \text {, } \\
& Y_{i j}, Z_{i j d t} \in\{0,1\} \quad \forall i, j, d, t \in T .
\end{aligned}
$$

The first objective function aims to minimize two terms as follows. The first term represents costs associated with caregivers' workload deviation from the average value. It balances workload among caregivers that in turn results in economic and behavioral benefits. The second term concerns training and maintenance costs. These two terms establish a tradeoff between the balancing cost (as operating cost) and cross-training cost. Factors $\pi_{1}$ and $\pi_{2}$ are weight factors, and setting them differently leads to different arguments. A higher value of $\pi_{1}$ indicates that the organization emphasizes workload balancing. On the other hand, cross-training costs may lead to higher settings for factor $\pi_{2}$. The second objective function attempts to minimize the number of employees for each service. Finally, the last objective function is aimed at maximizing caregiver satisfaction.

Eq. (3) calculate caregiver's workload deviation from the mean workload. Constraints (4) are related to regularity constraint. Constraints (5) guarantee that a caregiver is assigned to a given service only if he is trained for it. Constraints (6) force caregivers 
to be assigned to the services they have to perform. Eqs. (7)-(9) guarantee that the demand for different services (whether general, special, or synchronization constraint) is assigned to the caregivers. Constraints (10) prevent working hours per day from exceeding the maximum working time of each caregiver in each day. The maximum desired multi-functionality of each caregiver is represented by Constraints (11). The caregivers' satisfaction level is calculated through Constraints (12). Constraints (13) ensure that no service is assigned to absent caregivers. Finally, the domain of each decision variable is denoted by Constraints (14)(16).

The presented model can be simplified by linearizing the absolute term in the objective function. The nonlinear term:

$$
\left|\sum_{i}^{I} \operatorname{Time}_{i} \cdot X_{i j d t}-\tilde{X}_{d t}\right|,
$$

in the 1st objective function can be linearized by Eqs. (17) and Constraints (18). In addition, this nonlinear term is replaced by term $\left(\eta_{j d t}^{1}+\eta_{j d t}^{2}\right)$ :

$$
\begin{array}{lr}
\eta_{j d t}^{1}-\eta_{j d t}^{2}=\sum_{i}^{I} \operatorname{Time}_{i} . X_{i j d t}-\tilde{X}_{d t} & \forall j, d, t \in T, \\
\eta_{j d t}^{1}, \eta_{j d t}^{2} \geq 0 & \forall j, d, t \in T .
\end{array}
$$

\section{Solution methodology}

NSGA-II, which was first proposed by Deb et al. [38], is one of the most famous and applicable multi-objective evolutionary algorithms. This fame arises from its capability to cope with constraint as well as to promote diversity of solutions. The efficiency and convergence of the GA have been verified in the literature. In this algorithm, a non-dominance technique and a crowding distance are used to find a set of solutions ordered by fronts and select the population fronts. The pseudo-code of the proposed NSGA-II is shown in Algorithm 1. The main components of applied NSGAII are discussed in detail in the following subsections.

\subsection{Chromosome representation}

The first step in NSAG-II and many other metaheuristic algorithms is designing a suitable chromosome to present solutions and maintain the feasibility of the generated chromosomes. Each chromosome consists of a sequence of real, binary, or integer numbers (genes) and is represented by two approaches: direct and indirect encoding. In this study, the direct approach is used, meaning that a chromosome totally represents a solution. The designed chromosome is shown in Figure 1. In this representation, each gene stands for a service, and the chromosome shows which service is assigned to which caregiver at which time slot. Figure 1 indicates that service 3 is assigned to caregiver 2 at time slot 2 on day 1.

\subsection{Initialization}

A random population for initialization leads to the production of some infeasible solutions. To deal with this issue, a heuristic approach is proposed to generate feasible initial solutions. Who-rule is used to determine which caregiver to assign to services. In this rule, service is assigned to a caregiver according to the workloads of caregivers so that the workloads of

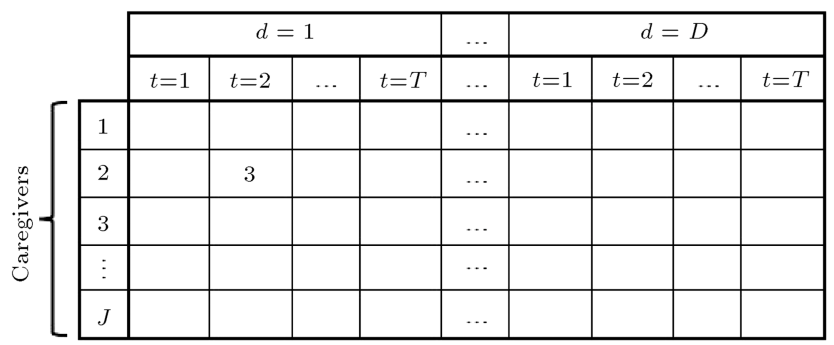

Figure 1. The chromosome representation for NSGA-II.

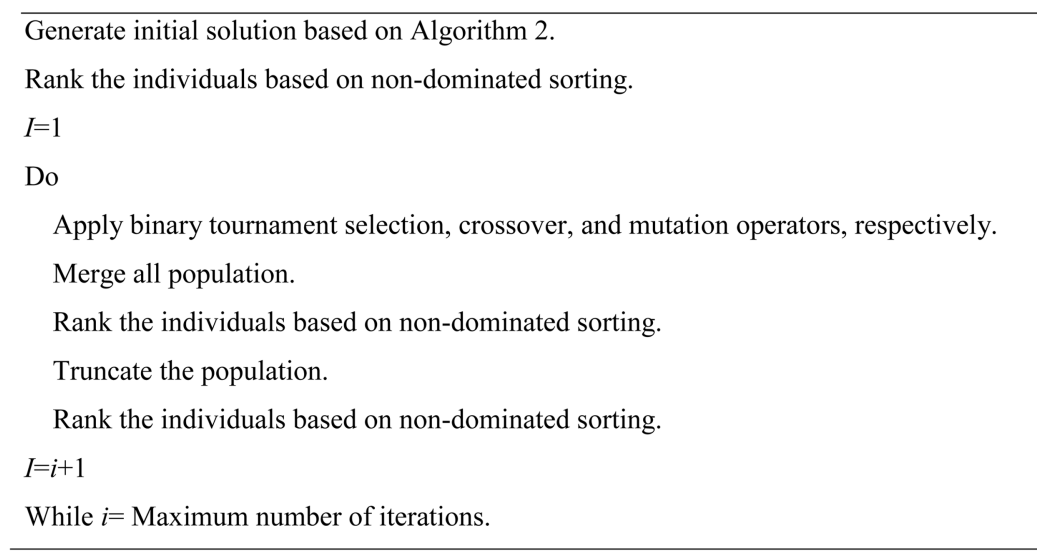

Algorithm 1. NSGA-II procedure. 


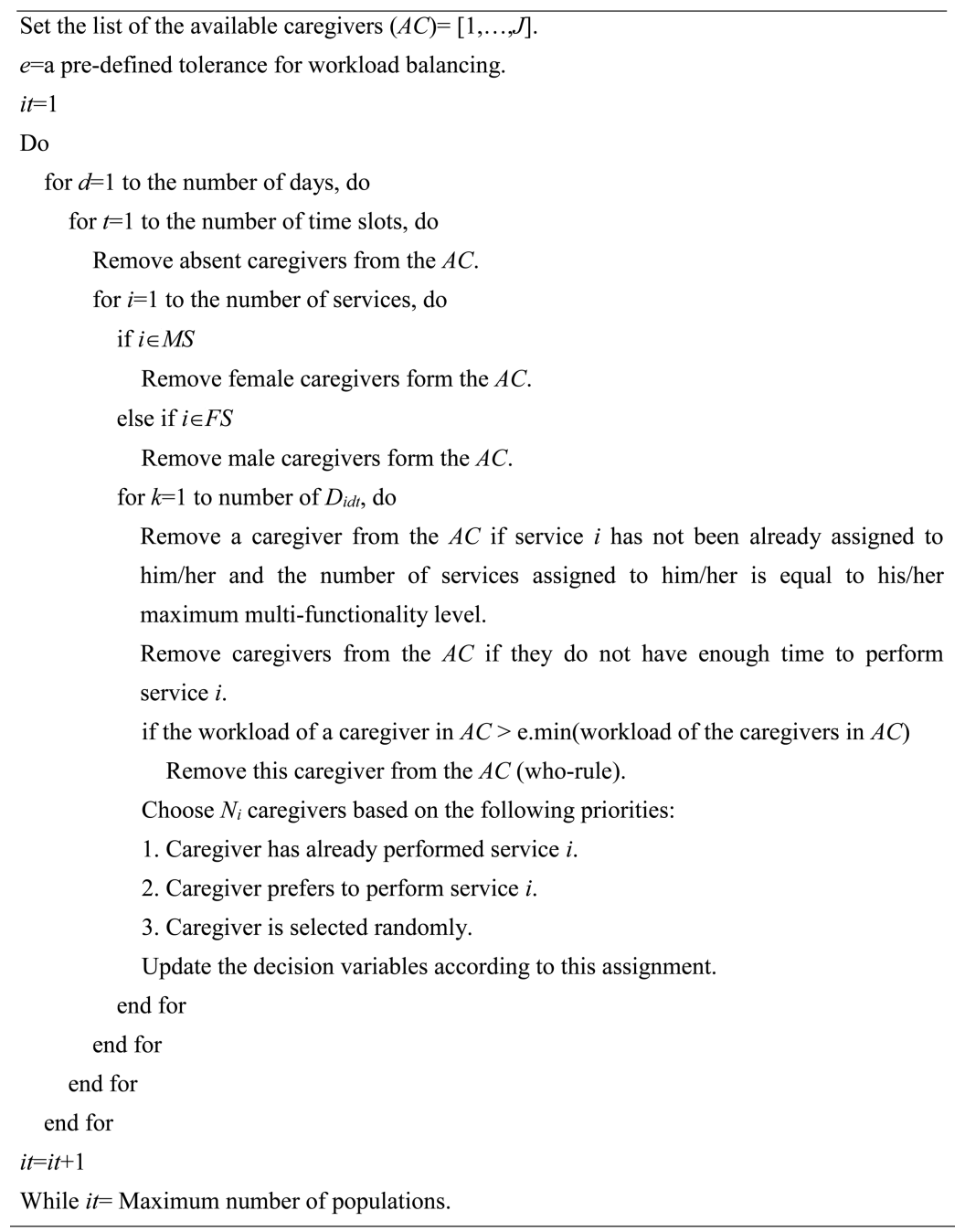

Algorithm 2. Heuristic procedure for initialization.

caregivers are balanced [39]. The pseudo-code of this approach is summarized in Algorithm 2.

\subsection{Non-dominated sorting}

The non-dominated sorting algorithm assigns a rank to each individual in the population based on nondominance before the selection strategy as well as after applying crossover and mutation operators [40]. To rank and select the fronts, a non-dominance technique and a crowding distance are applied. In a multiobjective model, solution $x$ dominates solution $y$ if:

1. $\mathrm{OFV}(x) \leq \mathrm{OFV}(y)$ for all objectives;

2. $\operatorname{OFV}(x)<\mathrm{OFV}(y)$ for at least one objective.

If a solution is not dominated by any other solution, it belongs to the front one. In addition, solutions in the front row are dominated only by solutions in the front one.

After classifying the solutions in different fronts, a crowding distance is used to estimate the density of solutions and rank the solutions in the same front. The crowding distance is computed as follows:

$$
d_{i}=\sum_{j=1}^{B} \frac{\left|f_{j}^{i+1}-f_{j}^{i-1}\right|}{\left|f_{j}^{\max }-f_{j}^{\min }\right|},
$$

where $B$ is the number of objective functions, $f_{j}^{i+1}$ is the $j$ th objective function of the $(i+1)$ th solution, $f_{j}^{i-1}$ is the $j$ th objective function of the $(i-1)$ th solution, and $f_{j}^{\max }$ and $f_{j}^{\mathrm{min}}$ are maximum and minimum values of the objective functions, respectively.

\subsection{Genetic operators}

The binary tournament selection procedure is adopted as the selection strategy in this paper. This strategy is based on the fitness value and selects two solutions of the population size based on the non-dominance technique and crowding distance.

\subsubsection{Crossover}

Crossover operator that combines two individuals from a population to produce new offspring is the main 


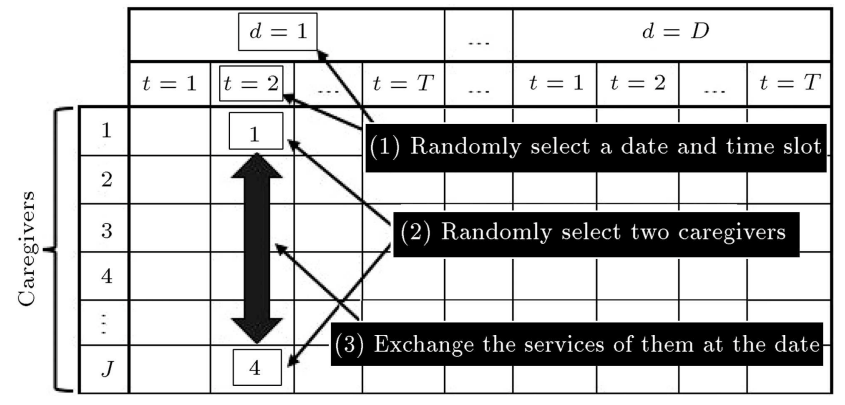

Figure 2. An example of employed mutation.

GA operator. In this study, two-point crossover is applied to produce new chromosomes. In two-point crossover, two crossing sections are selected randomly, and everything is swapped between the parents.

\subsubsection{Mutation}

Mutation is another GA operator that produces small random changes in chromosome to maintain the diversity of the population. Due to random changes in mutation, the solution becomes infeasible. Therefore, the mutation operator should keep the feasibility of the solutions. The basic operation of employed mutation is presented in the following. First, a day and time slot is selected randomly. Second, two caregivers are selected randomly. Finally, the services of these two positions are exchanged. Figure 2 represents an example of the employed mutation.

Some infeasible solutions may be produced by implementing crossover and mutation due to maximum working time and multi-functionality constraints. Here, to deal with this issue, the penalty function strategy is used and the values of constraint violations (Eqs. (20) and (21)) with proper penalty factors are added to the first objective function.

$$
\begin{aligned}
& \text { constraint violation } 1=\sum_{i=1}^{I} \operatorname{Time}_{i} \cdot E_{i j} \cdot X_{i j d t}-K \\
& \text { constraint violation } 2=\sum_{i=1}^{I} Y_{i j}-M_{j}
\end{aligned}
$$

\subsection{Parameter tuning}

Due to the significant effect of parameter values on the effectiveness of the algorithm, choosing appropriate parameters is necessary to preclude being trapped in local optimum and increase the search around interesting regions [41]. In this study, Taguchi Method (TM) is applied to obtain proper results and determine the best level of the parameters. TM is a robust approach of the factorial design of experiments, introduced by Genichi Taguchi. The aim of TM is to reduce the number of experiments and select a small set from all possible experiments. The NSGA-II parameters of
Table 3. NSGA-II parameters and their level values.

\begin{tabular}{lcccc}
\hline & \multicolumn{4}{c}{ Level } \\
\cline { 2 - 5 } Parameter & $\mathbf{1}$ & $\mathbf{2}$ & $\mathbf{3}$ & $\mathbf{4}$ \\
\hline Iteration (IT) & 100 & 200 & 300 & 400 \\
Population size (npop) & 100 & 150 & 200 & 250 \\
Tournament Size (TS) & 5 & 15 & 25 & 35 \\
Probability of crossover (pc) & 0.7 & 0.75 & 0.8 & 0.85 \\
Probability of mutation (pm) & 0.2 & 0.25 & 0.3 & 0.35 \\
\hline
\end{tabular}

iteration, population size, tournament size, crossover probability, and mutation probability are considered in the Taguchi's plan. These parameters and their level are shown in Table 3 .

\section{Computational results}

The behavior of the different elements and features of the proposed model is evaluated on a real-world HC provider called Dam organization, from which all required data are collected. This information relates to three adjacent districts of Tehran with the largest caregivers, and all the obtained results are solely concerned with this area. The planning horizon is a week ( 7 days), and about 1100 patients with 55 caregivers are assessed in this period. Table 4 presents the list of considered services in detail and also the demand for these services in the selected period. The information regarding demands is taken from the real historical data of the organization. $\mathrm{M}$ and $\mathrm{F}$ indicate male and female, respectively.

Table 5 provides information about the parameters of the model including training costs involved in learning how to perform a task. As mentioned before, these costs are zero for caregivers who are already qualified for a particular service. Moreover, the costs imposed by maintaining skills are considered to keep qualifications at a certain level. Table 5 also provides information about normalized visit time of services, indicating average visit time in a certain period as well as deviation from the average costs for workload balancing. All these parameters follow uniform distribution parameterized by specific $a$ 's and b's (e.g., $x \sim U(a, b))$.

\subsection{Taguchi execution}

In the proposed TM method, 16 experiments with 4 replications are conducted, and the algorithm is run under these designs. Figure 3 presents a graphical representation of the behavior of selected parameters according to their levels.

According to Figure 3, pc and npop have the most and the least ranges (the difference between the minimum and the maximum $S / N$ ratios) and, thus, have the most and the least influence on objective 
Table 4. List of the services and corresponding demand for them.

\begin{tabular}{clcc}
\hline No. & \multicolumn{1}{c}{ Service } & Caregiver & Demand \\
\hline 1 & Nursing & $\mathrm{M} / \mathrm{F}$ & 336 \\
2 & Paraclinic & $\mathrm{M} / \mathrm{F}$ & 121 \\
3 & Laser therapy & $\mathrm{F}$ & 12 \\
4 & Gynaecology nursing & $\mathrm{F}$ & 67 \\
5 & Physiotherapy & $\mathrm{M} / \mathrm{F}$ & 95 \\
6 & People with special diseases care (male) & $\mathrm{M}$ & 43 \\
7 & People with special diseases care (female) & $\mathrm{F}$ & 39 \\
8 & Elderly care (male) & $\mathrm{M}$ & 120 \\
9 & Elderly care (female) & $\mathrm{F}$ & 111 \\
10 & Child care & $\mathrm{M} / \mathrm{F}$ & 156 \\
\hline
\end{tabular}

Table 5. Parameters of the proposed model.

\begin{tabular}{|c|c|c|c|c|c|}
\hline No. & Service & $T C_{i j}\left(10^{4}\right)$ & $M C_{i j}\left(10^{4}\right)$ & $\operatorname{Time}_{i}(\mathrm{~h})$ & $C_{j t}\left(10^{4}\right)$ \\
\hline 1 & Nursing & {$[350500]$} & {$[4060]$} & {$\left[\begin{array}{ll}1 & 4\end{array}\right]$} & {$\left[\begin{array}{ll}250 & 400\end{array}\right]$} \\
\hline 2 & Paraclinic & {$\left[\begin{array}{lll}150 & 300\end{array}\right]$} & {$\left[\begin{array}{ll}20 & 40\end{array}\right]$} & {$\left[\begin{array}{ll}1 & 4\end{array}\right]$} & {$[250400]$} \\
\hline 3 & Laser therapy & {$\left[\begin{array}{lll}820 & 980\end{array}\right]$} & {$\left[\begin{array}{ll}70 & 85\end{array}\right]$} & {$\left[\begin{array}{ll}2 & 5\end{array}\right]$} & {$[250400]$} \\
\hline 4 & Gynaecology nursing & {$\left[\begin{array}{lll}480 & 630\end{array}\right]$} & {$\left[\begin{array}{ll}45 & 55\end{array}\right]$} & {$\left[\begin{array}{ll}1 & 4\end{array}\right]$} & {$\left[\begin{array}{ll}250 & 400\end{array}\right]$} \\
\hline 5 & Physiotherapy & {$\left[\begin{array}{lll}750 & 900\end{array}\right]$} & {$\left[\begin{array}{ll}60 & 75\end{array}\right]$} & {$\left[\begin{array}{ll}2 & 5\end{array}\right]$} & {$\left[\begin{array}{ll}250 & 400\end{array}\right]$} \\
\hline 6 & People with special diseases care (male) & {$\left[\begin{array}{ll}300 & 450\end{array}\right]$} & {$\left[\begin{array}{ll}45 & 60\end{array}\right]$} & {$\left[\begin{array}{ll}2 & 5\end{array}\right]$} & {$[250400]$} \\
\hline 7 & People with special diseases care (female) & {$\left[\begin{array}{ll}300 & 450\end{array}\right]$} & {$\left[\begin{array}{ll}45 & 60\end{array}\right]$} & {$\left[\begin{array}{ll}2 & 5\end{array}\right]$} & {$\left[\begin{array}{ll}250 & 400\end{array}\right]$} \\
\hline 8 & Elderly care (male) & {$\left[\begin{array}{ll}150 & 250\end{array}\right]$} & {$\left[\begin{array}{ll}10 & 20\end{array}\right]$} & {$\left[\begin{array}{ll}2 & 5\end{array}\right]$} & {$[250400]$} \\
\hline 9 & Elderly care (female) & {$\left[\begin{array}{ll}150 & 250\end{array}\right]$} & {$\left[\begin{array}{ll}10 & 20\end{array}\right]$} & {$\left[\begin{array}{ll}2 & 5\end{array}\right]$} & {$[250400]$} \\
\hline 10 & Child care & {$\left[\begin{array}{ll}150 & 250\end{array}\right]$} & {$\left[\begin{array}{ll}10 & 20\end{array}\right]$} & {$\left[\begin{array}{ll}2 & 5\end{array}\right]$} & {$\left[\begin{array}{ll}250 & 400\end{array}\right]$} \\
\hline
\end{tabular}

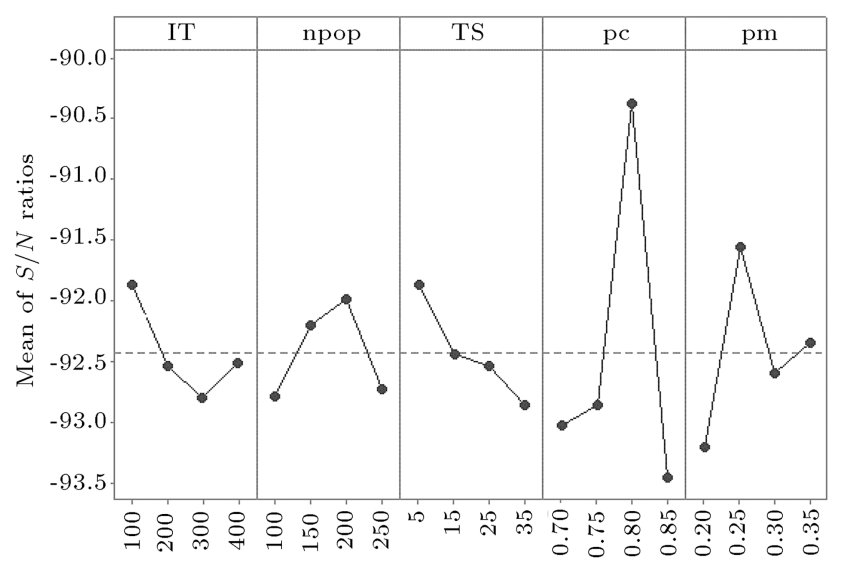

Figure 3. Main effects plot for $S / N$ ratios.

function value, respectively. In addition, the highest $S / N$ ratio is desired to achieve the minimum objective function value for each factor. The best values for parameters iteration, population size, tournament size, crossover probability, and mutation probability are 100 , $200,5,0.80$, and 0.25 , respectively.

\subsection{Comparative algorithm}

In this study, due to the absence of benchmark available in the literature, a non-dominated ranking genetic algorithm (NRGA) is used to validate the obtained results. The pseudo-code of NRGA is summarized in Algorithm 3.

Three comparison indexes are taken into consideration to validate the proposed NSGA-II. These indexes are defined as follows:

- Quality Index (QI): This index computes the percentage of the Pareto-optimal solutions for NSGAII and NRGA. A higher value is desirable for this index $[42,43]$;

- Mean Ideal Distance Index (MIDI): The distance between the best solutions and Pareto-optimal solutions. A lower value is desirable for this index [44];

- Spacing Index (SI): The distribution of the spread of the non-dominated set solutions. A lower value is desirable for this index [45-47].

In order to compare NSGA-II and NRGA, 25 test problems with different sizes are designed, as shown in Table 6. It should be noted that test problem (15) represents the actual size of the main problem related to the case study.

The results of comparing NSGA-II and NRGA based on the three comparison indexes for different test problems are shown in Table 7 . Note that each 


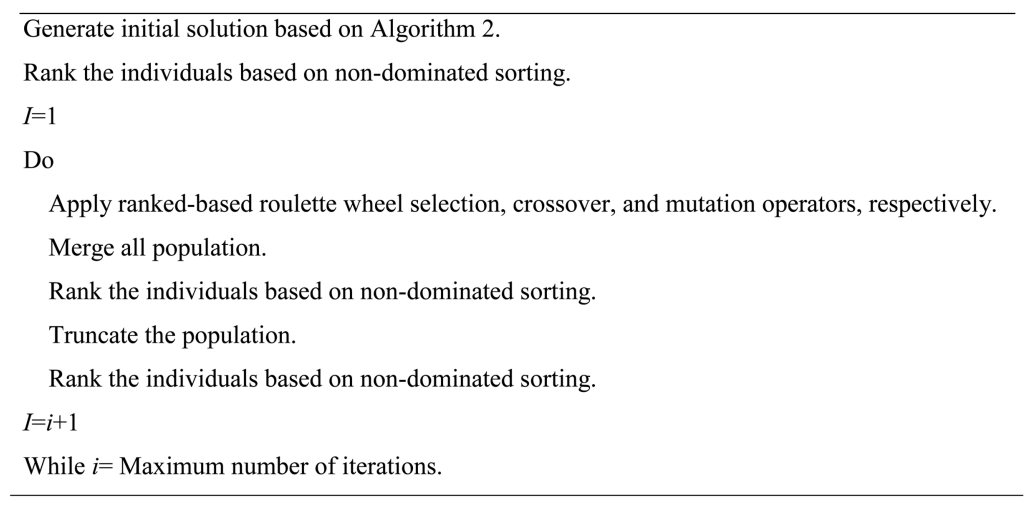

Algorithm 3. NRGA procedure.

Table 6. Data of the test problems.

\begin{tabular}{cccc}
\hline $\begin{array}{c}\text { Test problem } \\
\text { no. }\end{array}$ & $\begin{array}{c}\text { Number of } \\
\text { services }\end{array}$ & $\begin{array}{c}\text { Number of } \\
\text { caregivers }\end{array}$ & $\begin{array}{c}\text { planning } \\
\text { horizon }\end{array}$ \\
\hline 1 & 4 & 30 & 1 \\
2 & 4 & 35 & 1 \\
3 & 4 & 40 & 1 \\
4 & 4 & 40 & 3 \\
5 & 6 & 35 & 3 \\
6 & 6 & 40 & 3 \\
7 & 6 & 45 & 3 \\
8 & 6 & 45 & 5 \\
9 & 8 & 45 & 5 \\
10 & 8 & 50 & 5 \\
11 & 8 & 55 & 5 \\
12 & 8 & 55 & 7 \\
13 & 10 & 45 & 7 \\
14 & 10 & 50 & 7 \\
15 & 10 & 55 & 7 \\
16 & 12 & 50 & 7 \\
17 & 12 & 55 & 7 \\
18 & 12 & 60 & 7 \\
19 & 14 & 50 & 7 \\
20 & 14 & 55 & 7 \\
21 & 14 & 60 & 7 \\
22 & 16 & 55 & 7 \\
23 & 16 & 60 & 7 \\
24 & 16 & 65 & 7 \\
25 & 18 & 60 & 7 \\
\hline
\end{tabular}

algorithm has been run 30 times for each test problem, and the average of the obtained results is reported in Table 7. NSGA-II outperformed NRGA in terms of comparative indices QI and SI for all 25 test problems. Furthermore, paired $t$-test at the significance level of $\alpha=0.05$ is used to compare these two algorithms based on MIDI. The hypotheses of this test are as follows:

$$
\begin{cases}H_{0}: & \mu_{N S G A-I I}=\mu_{N R G A} \\ H_{1}: & \text { Otherwise }\end{cases}
$$

If the null hypothesis is not rejected, this means that there is no significant difference between the means of these two algorithms, in which case the algorithm with lower average results is selected as the desired algorithm (according to MIDI). On the other hand, rejection of the null hypothesis indicates that there is a significant difference between the means of the two algorithms, in which case in order to select the efficient algorithm according to MIDI, the following test is used:

$$
\begin{cases}H_{0}: & \mu_{N S G A-I I}=\mu_{N R G A} \\ H_{1}: & \mu_{N S G A-I I}<\mu_{N R G A}\end{cases}
$$

If the null hypothesis of the above test is rejected, it can be concluded that NSGA-II outperforms NRGA in terms of MIDI and, thus, is selected as the superior algorithm. Otherwise, the algorithm with lower average result is selected. The $p$-value of the paired $t$-test is equal to 0.006; therefore, the null hypothesis is rejected, meaning that there is no significant difference between NSGA-II and NRGA based on MIDI. In order to choose the superior algorithm, the second statistical hypothesis testing is used. The $p$-value for this test is equal to 0.003 according to which the null hypothesis is rejected; thus, NSGA-II is selected as the superior algorithm. Based on the obtained results, the proposed NSGA-II is superior to NRGA in terms of all comparison indexes (i.e., QI, MIDI, and SI) and, thus, is recommended for the proposed multi-objective homecare problem. The remainder of the analyses and results are based on the NSGA-II for the actual size of the case study.

\subsection{Results and discussion}

The model is run with different values of $\pi_{1}$ and $\pi_{2}$. Five Pareto-optimal solutions are selected randomly and are reported for objective function terms with different values of $\pi_{1}$ and $\pi_{2}$ in Table 8 . The values of different terms of objective functions, including workload balancing cost, training cost, the number of employees for each service (regularity), and satisfaction level, are presented in Table 8. In addition, Figures 
Table 7. Comparison results between the NSGA-II and NRGA based on to the comparison indexes.

\begin{tabular}{|c|c|c|c|c|c|c|}
\hline \multirow[b]{2}{*}{ problem no. } & \multicolumn{2}{|c|}{ QI } & \multicolumn{2}{|c|}{ MIDI } & \multicolumn{2}{|c|}{ SI } \\
\hline & NSGA-II & NRGA & NSGA-II & NRGA & NSGA-II & NRGA \\
\hline 1 & 0.78 & 0.64 & 0.262 & 0.485 & 0.267 & 0.411 \\
\hline 2 & 0.78 & 0.69 & 0.284 & 0.239 & 0.366 & 0.434 \\
\hline 3 & 1 & 1 & 0.415 & 0.554 & 0.204 & 0.471 \\
\hline 4 & 0.82 & 0.79 & 0.236 & 0.474 & 0.266 & 0.371 \\
\hline 5 & 0.85 & 0.75 & 0.197 & 0.656 & 0.468 & 0.490 \\
\hline 6 & 1 & 0.83 & 0.512 & 0.225 & 0.209 & 0.333 \\
\hline 7 & 0.72 & 0.63 & 0.298 & 0.268 & 0.225 & 0.340 \\
\hline 8 & 0.83 & 0.72 & 0.230 & 0.697 & 0.340 & 0.459 \\
\hline 9 & 0.87 & 0.8 & 0.259 & 0.243 & 0.318 & 0.379 \\
\hline 10 & 1 & 1 & 0.489 & 0.279 & 0.244 & 0.384 \\
\hline 11 & 0.90 & 0.75 & 0.362 & 0.598 & 0.373 & 0.475 \\
\hline 12 & 0.95 & 0.75 & 0.312 & 0.674 & 0.221 & 0.306 \\
\hline 13 & 1 & 1 & 0.228 & 0.234 & 0.308 & 0.331 \\
\hline 14 & 0.95 & 0.71 & 0.239 & 0.572 & 0.245 & 0.416 \\
\hline 15 & 1 & 0.75 & 0.346 & 0.652 & 0.295 & 0.340 \\
\hline 16 & 0.94 & 0.9 & 0.321 & 0.293 & 0.246 & 0.312 \\
\hline 17 & 0.84 & 0.89 & 0.443 & 0.429 & 0.235 & 0.31 \\
\hline 18 & 0.98 & 0.76 & 0.321 & 0.498 & 0.217 & 0.24 \\
\hline 19 & 0.79 & 0.84 & 0.358 & 0.374 & 0.389 & 0.299 \\
\hline 20 & 0.87 & 0.8 & 0.204 & 0.296 & 0.242 & 0.37 \\
\hline 21 & 0.9 & 0.75 & 0.486 & 0.602 & 0.246 & 0.329 \\
\hline 22 & 0.99 & 0.82 & 0.289 & 0.369 & 0.353 & 0.33 \\
\hline 23 & 1 & 0.79 & 0.303 & 0.216 & 0.228 & 0.291 \\
\hline 24 & 0.83 & 0.84 & 0.307 & 0.424 & 0.344 & 0.325 \\
\hline 25 & 1 & 0.91 & 0.419 & 0.507 & 0.312 & 0.389 \\
\hline Average & 0.9036 & 0.8044 & 0.3248 & 0.4343 & 0.2864 & 0.3654 \\
\hline
\end{tabular}

4 and 5 show the Pareto-optimal fronts for the first and third objective functions with different values of $\pi_{1}$ and $\pi_{2}$. In Table 8 and Figures 4 and 5, Type 1 means $\pi_{1}=1$ and $\pi_{2}=1$ and Type 2 means $\pi_{1}=10$ and $\pi_{2}=1$. As mentioned before, a higher level of $\pi_{1}$ indicates that the organizations focus on workload balancing, while cross-training costs may lead to higher value settings for factor $\pi_{2}$.

The results of the proposed model for selected Pareto-optimal solution (number 4 ) with $\pi_{1}=10$ and $\pi_{2}=1$ are given in Table 9 . Furthermore, Table 10 provides the results of analyzing caregivers and their workloads only for 10 cases for different values of weight factors.

Table 9 shows the number of caregivers as well as the cross-training cost associated with each service. Based on the results obtained, the highest training cost is related to service nursing, which also possesses the highest number of caregivers. Several conclusions can be drawn based on the results provided in Table 10 . First, the results reveal that the caregivers' workload is relatively balanced; thus, there is no bottleneck. 
Table 8. Objective function values for different values of $\pi_{1}$ and $\pi_{2}$.

\begin{tabular}{|c|c|c|c|c|c|c|c|c|}
\hline \multirow[b]{2}{*}{ No. } & \multicolumn{2}{|c|}{$\begin{array}{l}\text { Cross-training } \\
\text { cost }\left(10^{4}\right)\end{array}$} & \multicolumn{2}{|c|}{$\begin{array}{l}\text { Workload balancing } \\
\text { cost }\left(10^{4}\right)\end{array}$} & \multicolumn{2}{|c|}{ Regularity } & \multicolumn{2}{|c|}{$\begin{array}{c}\text { Satisfaction } \\
\text { level }\end{array}$} \\
\hline & Type 1 & Type 2 & Type 1 & Type 2 & Type 1 & Type 2 & Type 1 & Type 2 \\
\hline 1 & 19111 & 22294 & 11941 & 10309 & 71 & 83 & 34.83 & 33.68 \\
\hline 2 & 20313 & 25270 & 12961 & 11745 & 79 & 94 & 38.46 & 36.27 \\
\hline 3 & 21436 & 27071 & 13704 & 13543 & 83 & 101 & 41.15 & 39.86 \\
\hline 4 & 25914 & 30670 & 17069 & 14280 & 96 & 114 & 44.91 & 43 \\
\hline 5 & 27864 & 33277 & 19663 & 16083 & 103 & 123 & 48.24 & 46.53 \\
\hline
\end{tabular}

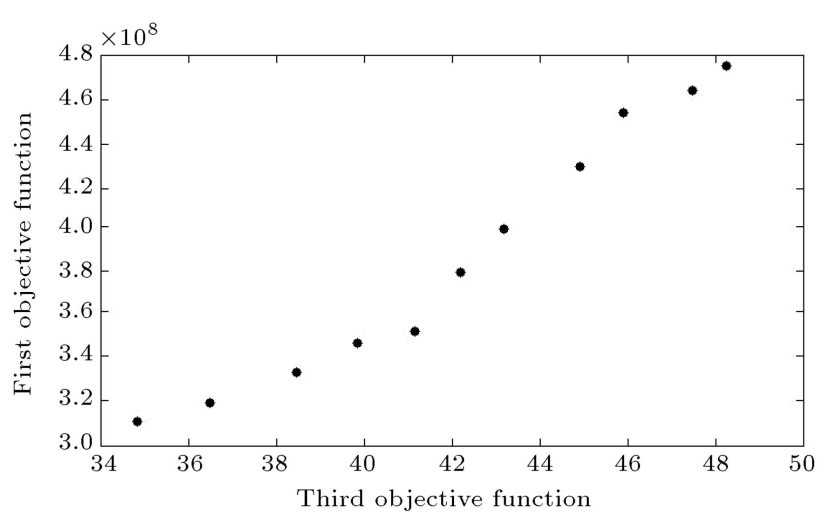

Figure 4. Pareto-optimal fronts for $\pi_{1}=1$ and $\pi_{2}=1$.

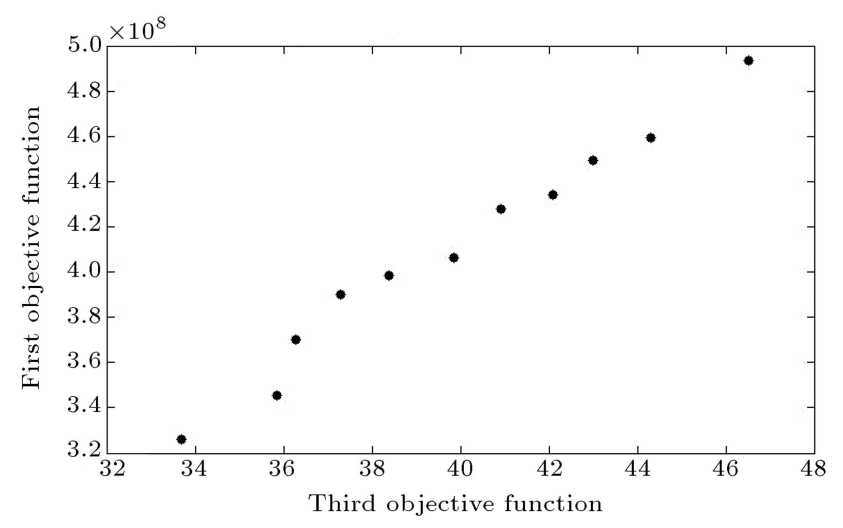

Figure 5. Pareto-optimal fronts for $\pi_{1}=10$ and $\pi_{2}=1$.

Second, in case workload balance is of considerable importance, it is possible to lay great emphasis on this issue by boosting the value of $\pi_{1}$. This leads to an increase in training cost. Third, when $\pi_{1}=10$, the caregivers' workload is more balanced (i.e., the caregivers' workload deviations from the average value are less) than when $\pi_{1}=1$. Fourth, it is realized that the caregivers' preferences are more satisfied and, except in a few cases, they were greater than 0.75. Finally, as the importance of the third objective function increases, caregivers' satisfaction level rises, too.

\section{Conclusions}

In this study, a multi-objective non-linear mathematical programming model was presented to address staff assignment problem considering cross-training of caregivers for $\mathrm{HC}$ services in healthcare systems. The first objective function aimed to minimize the costs associated with workload balancing, cross-training as well as maintenance, while the number of employees for each service was minimized in the second objective function. In addition, caregivers' satisfaction level was maximized in the third objective function. Moreover, several constraints including skill matching, staff preferences, regularity, synchronization, staff absenteeism, time capacity, and multi-functionality level were taken into account in building a service plan. Chaining concept was used to balance workloads of caregivers and allow shifting jobs among caregivers. Finally, due to the cultural conditions prevailing in Iran, it was supposed that services for men and women were separately performed. Dam homecare medical center organization located in Iran was considered as the intended case study; then, required data were gathered and the behavior of varied characteristics of the model was analyzed according to this case. A service plan was also built for 7 days (1 week), and the results determined which staff had to be cross-trained for each service and how the staff was assigned to services in each shift.

For the problem being NP-hard, a non-dominated sorting genetic algorithm (NSGA-II) with a proposed heuristic initialization procedure was applied, and the proposed model was solved based on a dataset collected from the proposed case. A who-rule was applied to determine which staff should be assigned to services. An NRGA and generated different test problems were used to validate the obtained results with respect to three comparison indexes including QI, MIDI, and SI. Based on the results obtained from statistical hypothesis tests (paired $t$-test), the proposed NSGAII was superior to NRGA in terms of all comparison indexes (i.e., QI, MIDI, and SI) and, thus, was recommended for the proposed multi-objective homecare problem. Analysis of caregivers showed that most of the caregivers achieved workload balance; thus, there was no bottleneck that could positively impact operational performance. Furthermore, when $\pi_{1}=$ 10 , the caregivers' workload was more balanced than when $\pi_{1}=1$. Finally, the caregivers' preferences 
Table 9. Analysis of the results of each service considering the number of caregivers and training costs.

\begin{tabular}{lccc}
\hline \multicolumn{1}{c}{ Service } & Caregiver & $\begin{array}{c}\text { Training-cost } \\
\left(\mathbf{1 0}^{\mathbf{4}}\right)\end{array}$ & Demand \\
\hline Nursing & 29 & 9046 & 336 \\
Paraclinic & 13 & 1854 & 121 \\
Laser therapy & 3 & 1779 & 12 \\
Gynaecology nursing & 6 & 2254 & 67 \\
Physiotherapy & 7 & 4945 & 95 \\
People with special diseases care (male) & 6 & 2089 & 43 \\
People with special diseases care (female) & 5 & 2023 & 39 \\
Elderly care (male) & 14 & 2234 & 120 \\
Elderly care (female) & 12 & 2069 & 111 \\
Child care & 19 & 2377 & 156 \\
\hline
\end{tabular}

Table 10. Analysis of caregivers and their workloads considering satisfaction level.

\begin{tabular}{|c|c|c|c|c|c|c|}
\hline \multirow[b]{2}{*}{ Caregiver } & \multicolumn{2}{|c|}{ Workload (h) } & \multicolumn{2}{|c|}{$\begin{array}{c}\text { Deviation from average } \\
\text { workload }(\mathrm{h})\end{array}$} & \multicolumn{2}{|c|}{ Satisfaction level } \\
\hline & Type 1 & Type 2 & Type 1 & Type 2 & Type 1 & Type 2 \\
\hline 1 & 33.805 & 39.2675 & 3.1375 & 1.2222 & 0.75 & 0.5 \\
\hline 2 & 34.95 & 37.16 & 1.9925 & 0.8852 & 1 & 1 \\
\hline 3 & 41.1625 & 37.89 & 4.22 & 0.1552 & 1 & 1 \\
\hline 4 & 33.155 & 35.68 & 3.7875 & 2.3652 & 0.75 & 0.75 \\
\hline 5 & 33.1025 & 37.9075 & 3.84 & 0.1377 & 0.66 & 0.66 \\
\hline 6 & 35.1 & 38.355 & 1.8425 & 0.3097 & 1 & 1 \\
\hline 7 & 36.9675 & 39.1825 & 0.025 & 1.1372 & 1 & 1 \\
\hline 8 & 39.25 & 38.17 & 2.3075 & 0.1247 & 1 & 0.75 \\
\hline 9 & 35.1125 & 36.0575 & 1.83 & 1.9877 & 0.75 & 0.66 \\
\hline 10 & 40.7825 & 39.46 & 3.84 & 1.4147 & 1 & 0.75 \\
\hline
\end{tabular}

were satisfied and, as the importance of the third objective function increases, caregivers' satisfaction level increased, too.

The distance between patient and caregivers is a major issue, which was not covered in this study. We believe combining cross-training with this issue would help researchers and organizations achieve a more real and precise service plan. Another issue that can affect organizations and can be of interest for researchers is uncertainty stemming from high variability of staff, material resources, etc. Furthermore, presenting an efficient algorithm that can handle all these issues particularly in various lengths of planning horizon could be another interesting topic.

\section{References}

1. Rasmussen, M.S., Justesen, T., Dohn, A., and Larsen, J. "The home care crew scheduling problem: Preference-based visit clustering and temporal dependencies", European Journal of Operational Research, 219(3), pp. 598-610 (2012).

2. Allaoua, H., Borne, S., Létocart, L., and Calvo, R.W. "A matheuristic approach for solving a home health care problem", Electronic Notes in Discrete Mathematics, 41, pp. 471-478 (2013).

3. Borsani, V., Matta, A., Beschi, G., and Sommaruga, F. "A home care scheduling model for human resources", in Service Systems and Service Management, 2006 International Conference on IEEE (2006). 
4. Benzarti, E., Sahin, E., and Dallery, Y. "Operations management applied to home care services: Analysis of the districting problem", Decision Support Systems, 55(2), pp. 587-598 (2013).

5. Lanzarone, E. and Matta, A. "Robust nurse-to-patient assignment in home care services to minimize overtimes under continuity of care", Operations Research for Health Care, 3(2), pp. 48-58 (2014).

6. Lanzarone, E. and Matta, A. "A cost assignment policy for home care patients", Flexible Services and Manufacturing Journal, 24(4), pp. 465-495 (2012).

7. Koeleman, P., Bhulai, S., and Van Meersbergen, M. "Optimal patient and personnel scheduling policies for care-at-home service facilities", European Journal of Operational Research, 219(3), pp. 557-563 (2012).

8. Denton, M., Brookman, C., Zeytinoglu, I., Plenderleith, J., and Barken, R. "Task shifting in the provision of home and social care in Ontario, Canada: implications for quality of care", Health \& Social Care in the Community, 23(5), pp. 485-492 (2015).

9. Rest, K.-D., Trautsamwieser, A., and Hirsch, P. "Trends and risks in home health care", Journal of Humanitarian Logistics and Supply Chain Management, 2(1), pp. 34-53 (2012).

10. Mutingi, M. and Mbohwa, C. "A fuzzy simulated evolution algorithm for multi-objective homecare worker scheduling", In Industrial Engineering and Engineering Management (IEEM), 2013 IEEE International Conference on, IEEE (2013).

11. Olivella, J., Corominas, A., and Pastor, R. "Task assignment considering cross-training goals and due dates", International Journal of Production Research, 51(3), pp. 952-962 (2013).

12. Hallgren, M. and Olhager, J. "Flexibility configurations: Empirical analysis of volume and product mix flexibility", Omega, 37(4), pp. 746-756 (2009).

13. Power, D. and Sohal, A.S. "Human resource management strategies and practices in just-in-time environments: Australian case study evidence", Technovation, 20(7), pp. 373-387 (2000).

14. Bidanda, B., Ariyawongrat, P., Needy, K.L., Norman, B.A., and Tharmmaphornphilas, W. "Human related issues in manufacturing cell design, implementation, and operation: a review and survey", Computers \& Industrial Engineering, 48(3), pp. 507-523 (2005).

15. Slomp, J., Bokhorst, J.A., and Molleman, E. "Crosstraining in a cellular manufacturing environment", Computers \& Industrial Engineering, 48(3), pp. 609$624(2005)$.

16. Slomp, J. and Molleman, E. "Cross-training policies and team performance", International Journal of Production Research, 40(5), pp. 1193-1219 (2002).
17. Marentette, K.A., Johnson, A.W., and Mills, L. "A measure of cross-training benefit versus job skill specialization", Computers \& Industrial Engineering, 57(3), pp. 937-940 (2009).

18. Wilke, H. and Meertens, R. "Group performance", International Series on Communication Skills (1994).

19. Bokhorst, J.A. and Slomp, J. "Design and operation of a cross-trained workforce", in Workforce Cross Training, D. Nembhard, Editor, pp. 3-63, CRC Press, Taylor \& Francis Group: Boca Raton, FL (2007).

20. Akjiratikarl, C., Yenradee, P., and Drake, P.R. "PSObased algorithm for home care worker scheduling in the UK", Computers \& Industrial Engineering, 53(4), pp. 559-583 (2007).

21. Hertz, A. and Lahrichi, N. "A patient assignment algorithm for home care services", Journal of the $O p$ erational Research Society, 60(4), pp. 481-495 (2009).

22. Rabeh, R., Saïd, K., and Eric, M. "Collaborative model for planning and scheduling caregivers' activities in homecare", In 18th IFAC World Congress (2011).

23. Gamst, M. and Jensen, T.S. "A branch-and-price algorithm for the long-term home care scheduling problem", In Operations Research Proceedings 2011, pp. 483-488, Springer (2012).

24. Liu, R., Xie, X., Augusto, V., and Rodriguez, C. "Heuristic algorithms for a vehicle routing problem with simultaneous delivery and pickup and time windows in home health care", European Journal of Operational Research, 230(3), pp. 475-486 (2013).

25. Mankowska, D.S., Meisel, F., and Bierwirth, C. "The home health care routing and scheduling problem with interdependent services", Health Care Management Science, 17(1), pp. 15-30 (2014).

26. Duque, P.M., Castro, M., Sörensen, K., and Goos, P. "Home care service planning. The case of Landelijke Thuiszorg", European Journal of Operational Research, 243(1), pp. 292-301 (2015).

27. Carello, G. and Lanzarone, E. "A cardinalityconstrained robust model for the assignment problem in home care services", European Journal of Operational Research, 236(2), pp. 748-762 (2014).

28. Mutingi, M. and Mbohwa, C. "A fuzzy particle swarm optimization approach for task assignment in home health care", In Industrial Engineering and Engineering Management (IEEM), 2013 IEEE International Conference on, IEEE (2013).

29. Mutingi, M. and Mbohwa, C. "Healthcare staff scheduling in a fuzzy environment: A fuzzy genetic algorithm approach", International Conference on Industrial Engineering and Operations Management (2014). 
30. Bokhorst, J., Slomp, J., and Gaalman, G. "On the who-rule in Dual Resource Constrained (DRC) manufacturing systems", International Journal of Production Research, 42(23), pp. 5049-5074 (2004a).

31. Bokhorst, J.A., Slomp, J., and Molleman, E. "Development and evaluation of cross-training policies for manufacturing teams", Iie Transactions, 36(10), pp. 969-984 (2004).

32. Yang, K.K. "A comparison of cross-training policies in different job shops", International Journal of Production Research, 45(6), pp. 1279-1295 (2007).

33. Yue, H., Slomp, J., Molleman, E., and Van Der Zee, D. "Worker flexibility in a parallel dual resource constrained job shop", International Journal of Production Research, 46(2), pp. 451-467 (2008).

34. Li, Q., Gong, J., Fung, R.Y., and Tang, J. "Multiobjective optimal cross-training configuration models for an assembly cell using non-dominated sorting genetic algorithm-II", International Journal of Computer Integrated Manufacturing, 25(11), pp. 981-995 (2012).

35. Liu, C., Yang, N., Li, W., Lian, J., Evans, S., and Yin, Y. "Training and assignment of multi-skilled workers for implementing seru production systems", The International Journal of Advanced Manufacturing Technology, 69(5-8), pp. 937-959 (2013).

36. Feng, Y. and Fan, W. "A system dynamics-based simulation model of production line with cross-trained workers", Journal of Statistical Computation and Simulation, 84(6), pp. 1190-1212 (2014).

37. Habibnejad, H., Rabbani, M., Javadi, B., and Ghorbani-Kutenaie, N. "A bi-objective mathematical model toward staff planning considering crosstraining", Journal of Industrial Engineering, 50(2), pp. 221-233 (2016).

38. Deb, K., Pratap, A., Agarwal, S., and Meyarivan, T. "A fast and elitist multiobjective genetic algorithm: NSGA-II", Evolutionary Computation, IEEE Transactions on, 6(2), pp. 182-197 (2002).

39. Bokhorst, J., Slomp, J., and Gaalman, G. "On the who-rule in Dual Resource Constrained (DRC) manufacturing systems", International Journal of Production Research, 42(23), pp. 5049-5074 (2004).

40. Bandyopadhyay, S. and Bhattacharya, R. "Solving a tri-objective supply chain problem with modified NSGA-II algorithm", Journal of Manufacturing Systems, 33(1), pp. 41-50 (2014).

41. Behnamian, J., Ghomi, S.F., and Zandieh, M. "A multi-phase covering Pareto-optimal front method to multi-objective scheduling in a realistic hybrid flowshop using a hybrid metaheuristic", Expert Systems with Applications, 36(8), pp. 11057-11069 (2009).
42. Moradi, H., Zandieh, M., and Mahdavi, I. "Nondominated ranked genetic algorithm for a multiobjective mixed-model assembly line sequencing problem", International Journal of Production Research, 49(12), pp. 3479-3499 (2011).

43. Mohammadi, M., Jolai, F., and TavakkoliMoghaddam, R. "Solving a new stochastic multi-mode p-hub covering location problem considering risk by a novel multi-objective algorithm", Applied Mathematical Modelling, 37(24), pp. 10053-10073 (2013).

44. Nekooghadirli, N., Tavakkoli-Moghaddam, R., Ghezavati, V., and Javanmard, S. "Solving a new bi-objective location-routing-inventory problem in a distribution network by meta-heuristics", Computers \& Industrial Engineering, 76, pp. 204-221 (2014).

45. Pasandideh, S.H.R., Niaki, S.T.A., and Asadi, K. "Biobjective optimization of a multi-product multi-period three-echelon supply chain problem under uncertain environments: NSGA-II and NRGA", Information Sciences, 292, pp. 57-74 (2015).

46. Idoumghar, L., Chérin, N., Siarry, P., Roche, R., and Miraoui, A. "Hybrid ICA-PSO algorithm for continuous optimization", Applied Mathematics and Computation, 219(24), pp. 11149-11170 (2013).

47. Attar, S., Mohammadi, M., Tavakkoli-Moghaddam, R., and Yaghoubi, S. "Solving a new multi-objective hybrid flexible flowshop problem with limited waiting times and machine-sequence-dependent set-up time constraints", International Journal of Computer Integrated Manufacturing, 27(5), pp. 450-469 (2014).

48. Trautsamwieser, A., Gronalt, M., and Hirsch, P. "Securing home health care in times of natural disasters", OR Spectrum, 33(3), pp. 787-813 (2011).

49. Stewart, B., Webster, D., Ahmad, S., and Matson, J. "Mathematical models for developing a flexible workforce", International Journal of Production Economics, 36(3), pp. 243-254 (1994).

50. Easton, F.F. "Cross-training performance in flexible labor scheduling environments", Iie Transactions, 43(8), pp. 589-603 (2011).

51. Kim, S. and Nembhard, D.A. "Rule mining for scheduling cross training with a heterogeneous workforce", International Journal of Production Research, 51(8), pp. 2281-2300 (2013).

52. Gnanlet, A. and Gilland, W.G. "Impact of productivity on cross-training configurations and optimal staffing decisions in hospitals", European Journal of Operational Research, 238(1), pp. 254-269 (2014).

53. Paul, J.A. and MacDonald, L. "Modeling the benefits of cross-training to address the nursing shortage", 
International Journal of Production Economics, 150, pp. 83-95 (2014).

54. Sammarco, M., Fruggiero, F., Neumann, W., and Lambiase, A. "Agent-based modelling of movement rules in DRC systems for volume flexibility: human factors and technical performance", International Journal of Production Research, 52(3), pp. 633-650 (2014).

\section{Biographies}

Hamed Habibnejad-Ledari obtained his MSc certificate from School of Industrial \& Systems Engineering, College of Engineering, University of Tehran. He received his BSc degree in Industrial Engineering from Babol University of Technology. His research interests include operation research, production planning and control, healthcare engineering, soft computing techniques, and scheduling.

Masoud Rabbani is a Professor at the School of Industrial Engineering and the Chairman of the Energy Management and Planning Research Institute at the University of Tehran, Iran. He received his $\mathrm{PhD}$ in Industrial Engineering at Amirkabir University of Technology, Tehran, Iran. His research interests include manufacturing and production systems, soft computing techniques, Multi-Criteria Decision-Making (MCDM), transportation, and scheduling. He has published in journals such as the International Journal of Advanced Manufacturing Technology, Applied Mathematics and Computation, International Journal of Production Research, Soft Computing, Advances in Engineering Software, Applied Mathematical Modeling, Engineering Optimization, International Journal of Manufacturing Technology and Management, Advanced Engineering Informatics, European Journal of Operational Research, Expert Systems with Applications, International Journal of Computer Mathematics, Applied Soft Computing, Computers and Operations Research, Journal of Manufacturing Systems, and Journal of Applied Sciences among others.

Nastaran Ghorbani-Kutenaie obtained her MSc certificate from the Department of Industrial Engineering, School of Engineering, University of Alzahra, Tehran, Iran. She received her BSc degree in Industrial Engineering from Babol University of Technology. Her research interests include healthcare engineering, performance assessment, customer relationship management, data envelopment analysis, and artificial intelligence. 